\title{
MEASURES AS CONVOLUTION OPERATORS ON HARDY AND LIPSCHITZ SPACES
}

\author{
BY MISHA ZAFRAN ${ }^{1}$ \\ Communicated by Alberto Calderón, November 11, 1974
}

In this note we announce some new results concerning the spectral theory of measures as convolution operators. To state our principal theorem, we introduce the following notation. If $X$ is a Banach space and $T$ is a bounded linear operator on $X$, we write $\operatorname{sp}(T, X)$ to denote the spectrum of $T$ on $X$. Let $G$ be an LCA group with dual group $\Gamma . M(G)$ will denote the class of finite regular Borel measures on $G$, and $M_{0}(G)=\{\mu \in M(G) \mid \hat{\mu}$ vanishes at infinity on $\Gamma$. For $\mu \in M(G)$, let $T_{\mu}$ denote the operator defined by $T_{\mu}(f)$ $=\mu * f$, that is, convolutions with $\mu$. Finally, let $H^{1}$ be the natural domain of the Hilbert transform on $L_{\mathbf{1}}(\mathbf{R})$, and let Lip $\alpha$ denote the usual class of bounded functions on $\mathbf{R}$ satisfying a Lipschitz condition of order $\alpha, 0<\alpha$ $<1$. We can now state our main result.

THEOREM 1. There exists a measure $\mu \in M_{0}(\mathbf{R})$ such that

(a) $\operatorname{sp}\left(T_{\mu}, H^{1}\right) \neq \hat{\mu}(\mathbf{R}) \cup\{0\}$, and

(b) $\operatorname{sp}\left(T_{\mu}, \operatorname{Lip} \alpha\right) \neq \hat{\mu}(\mathbf{R}) \cup\{0\}, 0<\alpha<1$.

This may be viewed as an analogue of the now classical Wiener-Pitt theorem concerning the invertibility of Fourier-Stieltjes transforms [4, Theorem 5.3.4]. Moreover, an elementary interpolation argument shows that if $1<$ $p<\infty$,

$$
\operatorname{sp}\left(T_{\nu}, L_{p}\right)=\hat{v}(\mathbf{R}) \cup\{0\},
$$

for all $\nu \in M_{0}(\mathbf{R})$ (see $[1, \S 1.4]$ ). Thus, in a sense, our theorem is intermediate between the $L_{1}$ and $L_{p}(1<p<\infty)$ cases.

The proof of Theorem 1 is based on the following result.

THEOREM 2. Let

AMS (MOS) subject classifications (1970). Primary 43A32, 47B99; Secondary $42 \mathrm{~A} 18$.

${ }^{1}$ This work was supported in part by NSF grant GP $28112 \mathrm{~A}$. 


$$
\psi_{\delta}(x)=\left\{\begin{aligned}
1 / \delta & \text { if } 0 \leqslant x<\delta / 2 \\
-1 / \delta & \text { if }-\delta / 2<x<0
\end{aligned}\right.
$$

Then there exists a measure $\nu \in M_{0}(\mathbf{R})$, of total variation norm 1 , which satisfies

$$
\lim _{\delta \rightarrow 0} \sup _{\delta}\left\|\nu_{\delta}\right\|_{1}=1
$$

for all $j=1,2, \cdots$.

The expression (*) arises since, as is readily verified,

$$
\limsup _{\delta \rightarrow 0}\left\|T\left(\psi_{\delta}\right)\right\|_{1} \leqslant C\|T\|_{O\left(H^{1}\right)}
$$

for every bounded linear operator $T$ on $H^{1}$. Here $C$ is an absolute constant and $\|T\|_{O\left(H^{1}\right)}$ denotes the operator norm of $T$ on $H^{1}$. Moreover, if $g \in$ $L_{1}(\mathbf{R}), \lim _{\delta \rightarrow 0}\left\|g * \psi_{\delta}\right\|_{1}=0$. Therefore, the expression $(*)$ provides us with a lower bound for the norms $\left\|\left(T_{\nu}-T_{g}\right)^{j}\right\|_{O\left(H^{1}\right)}, j=1,2, \ldots$, for every $g \in L_{1}(\mathbf{R})$. Consequently, since $\hat{v}$ vanishes at infinity, we have for appropriate $f \in L_{1}(\mathbf{R})$ that $\|\nu-f\|_{L_{\infty}(R)}<1$, whereas the spectral radius of the operator $T_{\nu-f}$ on $H^{1}$ is at least 1 .

A similar argument also applies to the space Lip $\alpha$ and certain of its variants, specifically, certain of the Taibleson spaces (see [5]). Thus Theorem 1 follows from Theorem 2 (with $\mu=\nu-f$ ).

The objects of study on Theorem 2 are measures of Cantor-Lebesgue type, which are subject to certain arithmetic constraints. Specifically, we examine infinite Bernoulli convolutions of the form

$$
\nu={ }_{k=1}^{\infty}\left(1 / 2 \delta_{0}+1 / 2 \delta_{t_{k}}\right)
$$

where the positive sequence $\left\{t_{k}\right\} \in l_{1}$ is chosen so that

(1) $t_{k+1} / t_{k} \rightarrow 0$ as $k \rightarrow \infty$, and $t_{n}>\Sigma_{k=n+1}^{\infty} t_{k}, n=1,2, \ldots$,

(2) $\hat{v}$ vanishes at infinity on $\mathbf{R}$, and

(3) $\left\{t_{k}\right\}$ is fully independent, that is, if $\left\{n_{k}\right\}$ is any bounded sequence of integers, and if $\Sigma_{k=1}^{\infty} n_{k} t_{k}=0$, then $n_{k}=0, k=1,2, \cdots$.

The existence of such sequences is guaranteed by probabilistic considerations (see [3, pp. 256-258]).

The proof of Theorem 2 then consists largely of a careful study of the $j$-fold sum of the Cantor set $\left\{\Sigma_{k=1}^{\infty} \epsilon_{k} t_{k} \mid \epsilon_{k}=0\right.$ or 1$\}$ generated by sequences 
$\left\{t_{k}\right\}$ of the above form. In particular, we show that the $j$-fold sum itself "looks like" a Cantor-type set which has been constructed in a "regular" way. We then integrate along the gaps arising at the various stages of the construction, to obtain estimate $(*)$ in Theorem 2 .

Finally, we remark that the techniques used here also yield the analogue of Theorem 1 for the circle group. Further results, detailed proofs, and some applications of this theory will appear in [6] .

\section{REFERENCES}

1. L. Hörmander, Estimates for translation invariant operators in $L^{p}$ spaces, Acta Math. 104 (1960), 93-140. MR 22 \#12389.

2. J.-P. Kahane and R. Salem, Ensembles parfaits et séries trigonometriques, Actualités Sci. Indust., no. 1301, Hermann, Paris, 1963. MR 28 \#3279.

3. Y. Meyer, Algebraic numbers and harmonic analysis, North-Holland, Amsterdam, 1972.

4. W. Rudin, Fourier analysis on groups, Interscience Tracts in Pure and Appl. Math., 12, Interscience, New York, 1962. MR 27 \#2808.

5. M. H. Taibleson, On the theory of Lipschitz spaces of distributions on Euclidean n-space. I. Principal properties; II, Translation invariant operators, duality, and interpolation, J. Math. Mech. 13 (1964), 407-479; ibid. 14 (1965), 821-839. MR 29 \#462; 31 \#5087.

6. M. Zafran, Measures as convolution operators on $H^{1}$ and Lip $\alpha$ (submitted).

DEPARTMENT OF MATHEMATICS, STANFORD UNIVERSITY, STANFORD, CALIFORNIA 94305 